\title{
Gas Chromatography-High Resolution Tandem Mass Spectrometry Using a GC-APPI-LIT Orbitrap for Complex Volatile Compounds Analysis
}

\author{
Young Jin Lee ${ }^{\text {a,b* }}$, Erica A. Smith ${ }^{\text {a,b }}$, and Ji Hyun Jun ${ }^{\text {a,b }}$ \\ ${ }^{a}$ Department of Chemistry, Iowa State University, Ames, IA 50011, USA \\ ${ }^{b}$ Ames Laboratory, US-DOE, Ames, IA 50011, USA
}

Received June 8, 2012; Revised June 17, 2012; Accepted June 17, 2012

First published on the web June 28, 2012; DOI: 10.5478/MSL.2012.3.2.29

\begin{abstract}
A new approach of volatile compounds analysis is proposed using a linear ion trap Orbitrap mass spectrometer coupled with gas chromatography through an atmospheric pressure photoionization interface. In the proposed GC-HRMS/MS approach, direct chemical composition analysis is made for the precursor ions in high resolution MS spectra and the structural identifications were made through the database search of high quality MS/MS spectra. Successful analysis of a complex perfume sample was demonstrated and compared with GC-EI-Q and GC-EI-TOF. The current approach is complementary to conventional GC-EI-MS analysis and can identify low abundance co-eluting compounds. Toluene co-sprayed as a dopant through API probe significantly enhanced ionization of certain compounds and reduced oxidation during the ionization.
\end{abstract}

Key words: Gas chromatography, Atmospheric pressure photoionization (APPI), Linear ion trap (LIT), Orbitrap, Volatile compounds

\section{Introduction}

GC-MS is an essential tool in chemical analysis of complex compounds and routinely used for environmental analysis, quality control, and drug testing. ${ }^{1}$ A quadrupole mass analyzer is the most popular detector in GC-MS, but its unit mass resolution hampers confident identification of unknown compounds. TOF MS as a GC detector has become popular in the last decade because of its fast speed and high mass accuracy. ${ }^{2}$ However, the mass accuracy is typically limited to $10 \mathrm{ppm}$ and not sufficient to uniquely define many chemical compositions. Higher resolution mass spectrometers, such as Fourier transform ion cyclotron resonance (FT ICR) ${ }^{3}$ and Orbitrap, ${ }^{4,5}$ have recently been used for the analysis of GC separated compounds and enabled unique chemical composition assignment.

Electron ionization (EI) is the most adopted ionization technique for GC-MS because of its non-specificity for most organic compounds and availability to search against an EI-MS spectral library. ${ }^{6}$ Extensive fragmentation in EI, however, often leads to the absence of molecular ions and difficulty in identifying co-eluting low abundance molecules. Soft ionization using chemical ionization and clear GC separation is necessary to overcome the limitations. In addition, an EI/CI source is not compatible with an ESI/API source designed for LC-MS and is not available in most

*Reprint requests to Young Jin Lee

E-mail: yjlee@iastate.edu high-end mass spectrometers.

Atmospheric pressure ionization (API) has been developed for GC-MS many decades ago, ${ }^{7}$ but has shown its usefulness only in negative ion mode for selective ionization of certain classes of compounds. ${ }^{8}$ Recent commercialization of highly sensitive API sources developed for LC-MS has re-vitalized its application for GC-MS and has been applied not only to quadrupole $\mathrm{MS}^{4}$ and TOF $\mathrm{MS}^{9-12}$ but also Q-TOF, ${ }^{13}$ FT ICR, ${ }^{3}$ and Orbitrap. ${ }^{4}$ However, mass spectrometric data acquisition methods have been limited to MS only scans. GC-MS/MS is often used for the analysis of complex volatile compounds, but mostly with chemical ionization and using low resolution tandem mass spectrometers such as ion trap or triple quadrupole MS. ${ }^{14}$

Here, we report the development of a gas chromatography high resolution tandem mass spectrometry (GCHRMS/MS) approach using a GC-APPI-linear ion trap (LIT) Orbitrap mass spectrometer for the analysis of complex volatile compounds. We are taking an approach similar to that of LC-MS/MS, particularly those commonly adopted for high throughput proteome analysis. ${ }^{15}$ This includes automatic MS/MS with LIT for precursors selected from the preview scan of Orbitrap and dynamic exclusion of previously acquired precursor ions for MS/MS of low abundance ions. Our data analysis protocol includes direct elemental composition analysis of precursor ions followed by an MS/MS database search, which has potential to become high throughput but is currently limited by the database. The developed approach is applied for the analysis of a perfume sample and compared to the tradi- 
tional approach by GC-EI-Q and GC-EI-TOF. The utility of toluene as a dopant was also studied by co-spraying into the interface during GC-APPI-MS analysis.

\section{Experimental}

\section{Materials}

A perfume sample (CK One; Calvin Klein) was purchased from a local store and solvents were purchased from Sigma (St. Louis, MO) for the best purity (Chromasolv grade). Ultra high purity $(99.999 \%)$ helium and nitrogen gases were used for GC, GC-MS interface, and mass spectrometer.

\section{Gas chromatography and GC-MS interface}

The gas chromatograph used was a Varian (Walnut Creek, CA) Star 3400 CX GC with an HP-5MS column $(30 \mathrm{~m} \times 250 \mu \mathrm{m}, 0.25 \mu \mathrm{m}$ film thickness; J\&W Scientific, Folsom, CA). Original FID detector was removed and the port was then packed with insulation. A new port was made on the side of the GC oven to deliver the fused silica capillary to the mass spectrometer inlet via a heated transfer line. This was achieved by feeding the capillary through a tfitting to introduce heated sheath gas (nitrogen) that kept the capillary at a uniform temperature from the GC oven to the exit tip (Figure 1). The nitrogen gas was heated in the GC oven in stainless steel tubing before entering the tfitting with a flow rate of about $20 \mathrm{~mL} / \mathrm{min}$. This design is based off of McEwen's GC-APCI-MS interface, ${ }^{9}$ except that a home-made GC-MS interface with a glass rod and heating tape was used. The temperature of the glass rod was monitored with a thermocouple and controlled to maintain $280{ }^{\circ} \mathrm{C}$ using a temperature controller (HTS/Amptek, Stafford, TX). The front glass window of the Ion MAX source was removed to allow the heated glass rod/capillary to be interfaced to the mass spectrometer inlet (See the photos in Supplementary Figure 1). The distance between the end of the column and the mass spectrometer inlet is about $1 \mathrm{~cm}$, through which GC eluents are exposed to UV

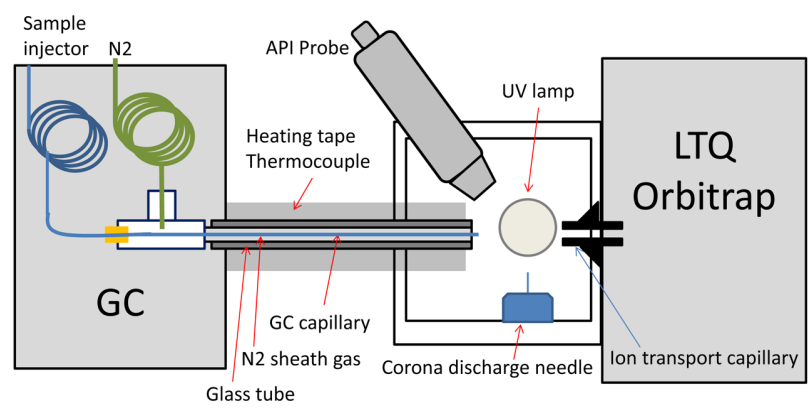

Figure 1. A schematic diagram of GC-API-LIT Orbitrap interface viewed from the side. Corona discharge needle is shown at the bottom for convenience, but it is at the same position with UV lamp out of the plane. photons produced by a Krypton lamp (photon energy of $10.0 \mathrm{eV}$ and $10.6 \mathrm{eV}$; Syagen, Tustin, CA) before being injected to the mass spectrometer.

The column was conditioned by flowing helium carrier gas overnight at a temperature of $250{ }^{\circ} \mathrm{C}$. Carrier gas flow rate was pressure controlled and the initial rate was estimated to be $4.7 \mathrm{~mL} / \mathrm{min}$. A splitless injection was used to introduce $1 \mu \mathrm{L}$ of the perfume sample to the injection port, which was set at $240{ }^{\circ} \mathrm{C}$. The GC oven temperature gradient started at $40^{\circ} \mathrm{C}$, was held for 2 minutes, then heated to $140{ }^{\circ} \mathrm{C}$ at $10{ }^{\circ} \mathrm{C} / \mathrm{min}$, then to $260{ }^{\circ} \mathrm{C}$ at $20{ }^{\circ} \mathrm{C} / \mathrm{min}$, and finally held at $260{ }^{\circ} \mathrm{C}$ for 7 minutes. The interface temperature was maintained at $280{ }^{\circ} \mathrm{C}$ for the duration of the run.

A dopant spray experiment was also performed by spraying dopant through the API probe to the GC-MS interface while the GC-MS experiment is being performed. A solvent mixture of $15 \%$ toluene and $85 \%$ methanol was used at a flow rate of $10 \mu \mathrm{L} / \mathrm{min}$ and a vaporization temperature of $350{ }^{\circ} \mathrm{C}$.

\section{Mass spectrometry}

An LTQ Orbitrap Discovery (Thermo Scientific, San Jose, CA) was used for the experiment with an Ion MAX APCI/APPI dual probe source. The ion transfer capillary temperature was set to $150{ }^{\circ} \mathrm{C}$. The low tube lens voltage of 7 volts was used to minimize in-source CID. Instrument parameters for the API probe such as sheath, auxiliary, and sweep gas were placed to their lowest allowed values except for dopant spray experiment. The instrument was calibrated nine days before the experiments and used without any further mass calibration.

GC-MS/MS data sets were acquired with Xcalibur software in the similar fashion for a typical LC-MS/MS acquisition. Namely, a high mass resolution Orbitrap data acquisition (nominal resolution at 30,000) is followed by one or two data dependent MS/MS scans in the linear ion trap. Preview FFT mode allows maximization of the duty cycle; an intermediate Orbitrap spectrum is interpreted onthe-fly for data dependent decision. Automatic gain control (AGC) was set at the target value of $2 \times 10^{5}$ ions for Orbitrap MS and $1 \times 10^{4}$ ions for LIT MS/MS. Dynamic exclusion was used for the duration of 30 seconds if MS/ MS spectra were acquired twice in 15 seconds. Background peaks are added to the reject mass list with the mass tolerance of $\pm 0.03 \mathrm{Da}$. Collision induced dissociation (CID) was used for MS/MS with a precursor isolation width of $2.0 \mathrm{Da}$ and collision energy of $50 \%$. The activation time was set to $30 \mathrm{~ms}$ with an activation Q-value of 0.250 .

\section{GC-EI-MS}

The same perfume sample was also analyzed on GC-EI-Q (quadrupole $\mathrm{MS}$ ) and GC-EI-TOF instruments for comparison. For GC-EI-Q, Agilent GC $(6890 \mathrm{~N})$ and MS $(5973 \mathrm{~N})$ were used with an HB-5MS column. For GC-EI- 


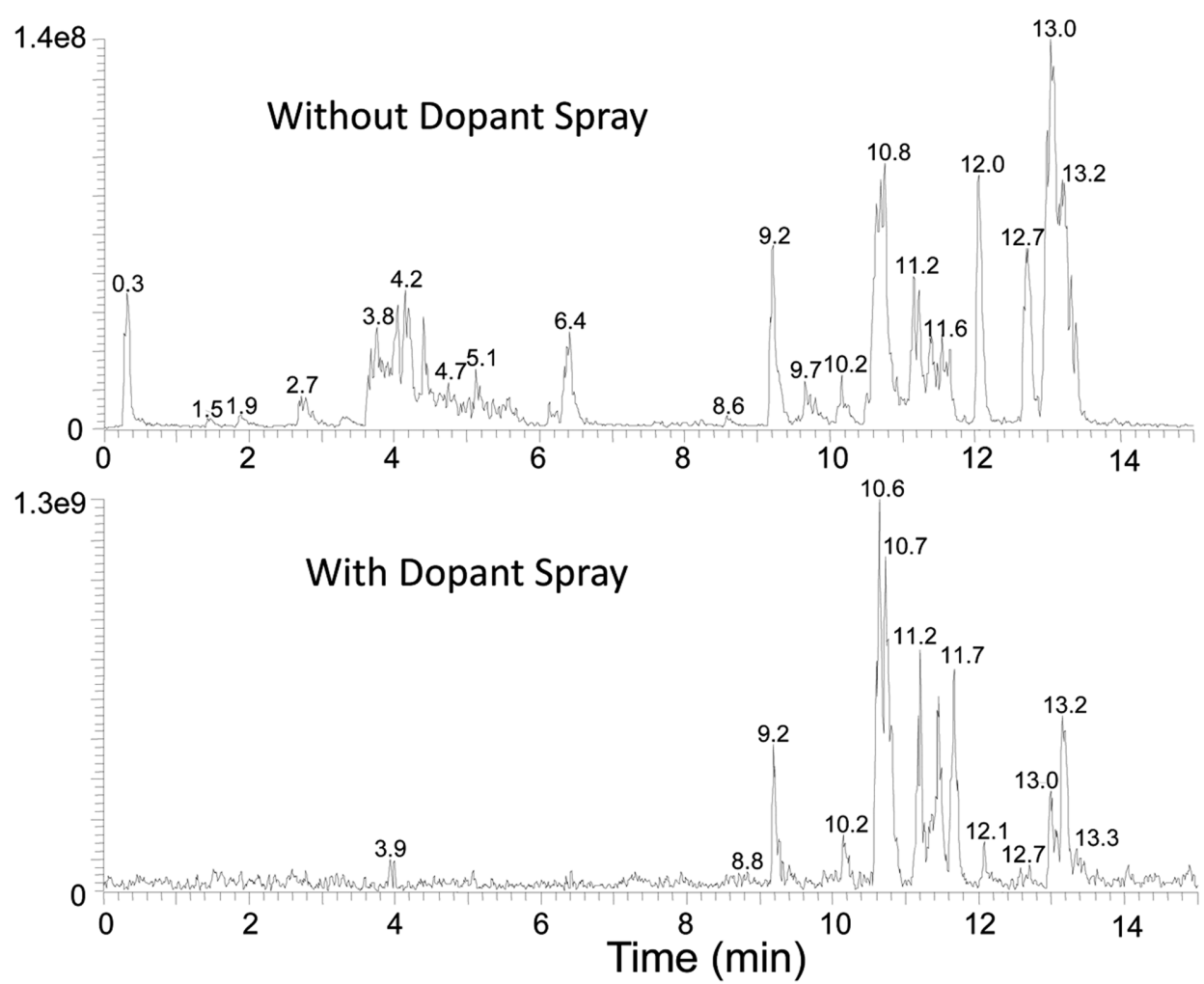

Figure 2. Base ion chromatograms of GC-APPI-LIT Orbitrap data of a perfume sample without (Top) and with (Bottom) dopant spray.

TOF, Agilent GC (6890C) and Micromass TOF (GTC) were used with a DB-5 column $(30 \mathrm{~m} \times 250 \mu \mathrm{m}, 0.25 \mu \mathrm{m}$ film thickness). Gas flow was controlled at a constant flow rate of $1 \mathrm{~mL} / \mathrm{min}$ for both instruments. A $1 \mu \mathrm{L}$ injection of the sample was used with the split ratio of 1:50 and 1:100 for GC-EI-Q and GC-EI-TOF, respectively. The same injector and oven temperature program was used with that in the GC-APPI-LIT Orbitrap experiment. EI of $70 \mathrm{eV}$ and a scan range of $m / z 35-650$ were used for both instruments and the scan rate was 0.42 and $0.4 \mathrm{sec} / \mathrm{scan}$ for GC-EI-Q and GC-EI-TOF, respectively. The TOF we used has limited dynamic range due to: 1) saturation of high ion currents in time-to-digital converter (TDC) detector system, and 2) ion suppression from co-injected lock-mass calibrants. To minimize the problems, the perfume sample was analyzed four times on GC-EI-TOF; a neat sample with and without lock-mass calibration, as well as a 20 times diluted sample with and without lock-mass calibration. Lock-mass calibration was used with single point calibration using 2,4,6-tris(trifluoromethyl)-1,3,5triazine at $\mathrm{m} / \mathrm{z} 284.9943$.

AMDIS software (NIST, v2.69) was used for automatic deconvolution and data analysis for GC-EI-Q data and MassLynx software (Micromass, v4.0) was used for GCEI-TOF with manual background removal. Tentative identification was made by searching EI spectra against the NIST08 EI-MS spectral library with the minimum match score of 800 . In GC-EI-TOF, tentative assignment was accepted for each chromatographic peak if the score is higher than 800 in any of the four data sets. The least mass error was accepted between the two lock-mass calibrated GC-EI-TOF data sets because mass error becomes significant in TOF MS when ion signal is too high $\left(\geq 10^{4}\right.$ ions) or too low ( $\leq 100$ ions).

\section{Results and Discussion}

\section{Overview of GC-API-LIT orbitrap}

Figure 1 shows the schematic diagram of GC-API-LIT Orbitrap interface and Supplementary Figure 1 shows the photos at the interface. The API interface designed by McEwen was adopted for the current study. ${ }^{9}$ The only major difference is that Thermo Finnigan's Ion MAX source allows simultaneous operation of APPI and APCI. We used only APPI in the current study but the use of APCI or both will be investigated in the future. The detailed description of the interface refers to the original paper by McEwen or a short summary in the experimental section.

Figure 2 shows the base ion chromatograms of a perfume sample analyzed by GC-APPI-LIT Orbitrap with and without dopant spray. A solution of $15 \%$ toluene in methanol was sprayed through the API probe in the dopant experiment to study its efficacy in enhancing ionization efficiency. Ion signals for early eluting peaks, Rt $<8 \mathrm{~min}$, 
are greatly reduced while late eluting peaks have an order of magnitude signal improvement overall. GC-HRMS/MS data were acquired using a similar strategy commonly adopted for LC-MS/MS: 1) MS acquisition in Orbitrap with the nominal mass resolution of 30,000 (defined at $\mathrm{m} / \mathrm{z} 400$ ); 2) one or two MS/MS acquisitions in LIT for the highest abundance ion(s) in the preview Orbitrap spectra; 3) dynamic exclusion of the previously acquired precursor ions in acquiring MS/MS for the next thirty seconds; 4) exclusion of common contamination peaks for MS/MS.

Fast MS scan speed is one of the most important requirements in GC-MS because a sufficient number of data points is needed across a very narrow chromatographic peak profile. The scan speed in GC-APPI-LIT Orbitrap was $0.66 \mathrm{sec}$ for a set of an Orbitrap scan and an MS/MS scan and $0.94 \mathrm{sec}$ for an Orbitrap scan and two MS/MS scans. In MS only mode, the scan speed was as fast as $0.188 \mathrm{sec}$ per scan at the nominal mass resolution of 7,500. Typical mass accuracy was below $3 \mathrm{ppm}$, even at the nominal mass resolution of 7,500, because Orbitrap provides higher mass resolution for the low masses commonly analyzed by GCMS; 17,000-10,000 for $m / z 100-300$ at nominal resolution of 7,500. The rest of the results and discussions will be restricted to the data sets shown in Figure 2, which were acquired with one Orbitrap scan and one LIT MS/MS scan in each acquisition cycle.

The home-made glass tube interface adopted in the current study has a few limitations. Among others, it produces significant contaminations. Major interference peaks are $m / z 279.1594,223.0969,205.0860$, and 149.0235

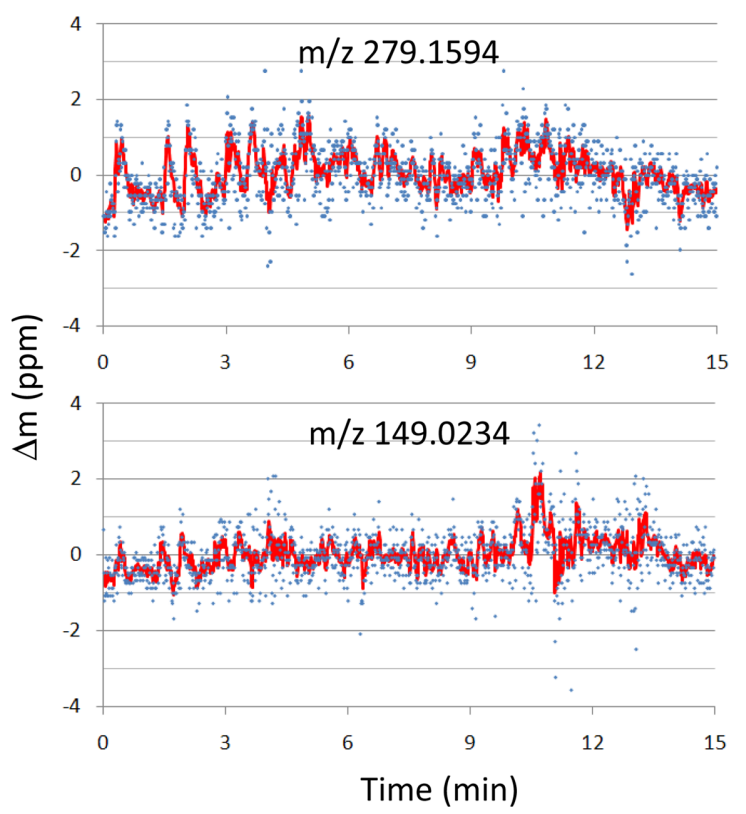

Figure 3. Mass precision of two contamination peaks. Blue dots indicate the peak position deviation from the mean value and red solid line is after five data point average. with relative abundances of $43,68,12$, and $13 \%$, respectively, in an MS spectrum averaged for retention time of 1.4-13.5 min (data not shown). These contaminations are present with a relative abundance of at least $0.5 \%$ at any retention time. The three most abundant contaminations are attributed to protonated plasticizers, presumably coming from the heating tape; diisobutylphthalate $\left(\left[\mathrm{C}_{16} \mathrm{H}_{22} \mathrm{O}_{4}+\mathrm{H}^{+}\right]\right.$; $\left.(\mathrm{m} / \mathrm{z})_{\text {cal }}=279.15909, \quad \Delta \mathrm{m}=1.1 \mathrm{ppm}\right), \quad$ diethylphthalate $\left(\left[\mathrm{C}_{12} \mathrm{H}_{14} \mathrm{O}_{4}+\mathrm{H}^{+}\right] ;(\mathrm{m} / \mathrm{z})_{\mathrm{cal}}=223.09649, \Delta \mathrm{m}=1.9 \mathrm{ppm}\right)$, and phthalic anhydride $\left(\left[\mathrm{C}_{8} \mathrm{H}_{4} \mathrm{O}_{3}+\mathrm{H}^{+}\right] ;(\mathrm{m} / \mathrm{z})_{\text {cal }}=149.02332\right.$, $\Delta \mathrm{m}=0.9 \mathrm{ppm})$. Since these ions are present throughout the chromatographic separation, they can be good indicators of mass position reproducibility and can be used for internal calibration.

Figure 3 shows the mass position fluctuations of two contamination peaks, $\mathrm{m} / \mathrm{z} 279.1594$ and $\mathrm{m} / \mathrm{z}$ 149.0234, over the duration of $15 \mathrm{~min}$ chromatographic separation. The maximum deviation is less than \pm 3 ppm and RMS deviation is $0.75 \mathrm{ppm}$ for $\mathrm{m} / \mathrm{z} 279.1594$ and the maximum deviation is $\pm 3.5 \mathrm{ppm}$ and RMS deviation is $0.67 \mathrm{ppm}$ for $\mathrm{m} / \mathrm{z}$ 149.0234. The maximum deviation is larger for $\mathrm{m} / \mathrm{z}$ 149.0234 because of its lower S/N (20 30 compared to $70 \sim 100$ for $m / z 279.1594$ ); its position is greatly affected by the total ion flux at the given retention time. Slightly higher RMS deviation for $m / z 279.1594$ might have come from its lower mass resolution (40,700 compared to 55,700 for $\mathrm{m} / \mathrm{z}$ 149.0234). Averaging a few MS spectra over the chromatographic peak profile enhances reproducibility and mass accuracy. The solid lines in Figure 3 shows the mass value fluctuations after a five point data average, corresponding to $\sim 3$ second-wide chromatographic peak. The maximum deviation is now $+1.6 /-1.4 \mathrm{ppm}$ and $+2.2 /-1.1 \mathrm{ppm}$ for $\mathrm{m} / \mathrm{z}$ 279.1594 and $m / z$ 149.0234, respectively. RMS deviation is also reduced to $0.53 \mathrm{ppm}$ and $0.43 \mathrm{ppm}$, respectively. All the peaks used in the subsequent data analysis had a $\mathrm{S} / \mathrm{N}$ ratio much higher than that of $m / z$ 149.0234. Hence, after averaging a few MS spectra, the mass accuracy is expected to be within $\sim 2$ ppm with internal calibration and $\sim 4 \mathrm{ppm}$ with external calibration only.

The precision of mass peak position is affected by the change in incoming ion flux at any given time in chromatography-mass spectrometry. When ion flux is low, mass precision is low because of insufficient ion statistics (low $\mathrm{S} / \mathrm{N}$ ). On the other hand, when it is too high, peak position is affected by the space-charge effect (Coulomb repulsion between ions). While Orbitrap and FTICR provides reliable mass precisions over the wide range of ion flux (RMS mass accuracy below 2 ppm for ion flux change of $\left.\sim 10^{4}\right),{ }^{16} \mathrm{TOF} \mathrm{MS}$, commonly used with GC, has a rather narrow range of acceptable ion flux in order to maintain good mass accuracy (RMS mass accuracy below 3 ppm for ion flux change of $\sim 100$, or $5 \mathrm{ppm}$ for ion flux change of $\left.\sim 10^{3}\right) .{ }^{17}$ Hence, internal calibration with a co-sprayed standard compound is often necessary in TOF MS in order to maintain high mass accuracy throughout the chromato- 
graphic separation. High mass resolution and stable ion flux controlled by AGC (automatic gain control) allow us to maintain high mass accuracy in our GC-APPI-LIT-Orbitrap throughout the chromatographic time scale, even without any internal calibration.

\section{Data analysis for GC-HRMS/MS}

We propose a potentially high throughput data analysis protocol for the data set obtained with GC-HRMS/MS. The main idea is similar to that of common proteomics data analysis; extraction of all the MS/MS spectra with accurate precursor mass information and MS/MS database search for identification. The major difference is we also perform direct chemical composition analysis, which was enabled because unique chemical composition assignment is possible for low mass ions with accurate mass information. This approach, along with MS/MS data acquisition in dynamic exclusion mode, can potentially identify hundreds of compounds in a single data set as shown routinely in typical LC-MS/MS based proteomics. For example, MASCOT distiller (v. 2.3.2.0; Matrix Science, UK) could extract over six hundred high quality MS/MS spectra along with their accurate precursor ion information for the data set shown in Figure 2.

High throughput application of the proposed protocol is currently overshadowed by a few practical limitations. First, the precursor spectrum is composed of not only molecular or pseudo-molecular ions but also oxidative primary ions and some in-source CID fragments, as will be discussed in the next section. Further optimization of experimental parameters is necessary to minimize this problem. Second, there is no comprehensive MS/MS database or a priori prediction of MS/MS spectra currently available. Publicly available databases, such as NIST MS/ MS database, Metlin, and MassBank, only have a limited number of entries. There are some efforts for $a b$ initio interpretation of MS/MS spectra; ${ }^{18,19}$ however, they are not comprehensive and their wide-spread use is limited. In the proteomics data analysis pipeline, in contrast, fragmentation of peptides is rather predictable, as they mostly occur through amide backbone cleavage. Once these bottlenecks are overcome, a high throughput data analysis program could be written that automatically calculates each chemical composition of precursor ions and searches the MS/MS spectra against either comprehensive database or theoretically predicted MS/MS spectra of all the possible structural isomers.

In the current study, we demonstrate the plausibility of the proposed approach by manually analyzing a few high quality MS and MS/MS spectra. The process was divided into two steps: chemical composition analysis of a few major peaks and their MS/MS search against public MS/ MS databases. Twenty six major peaks were chosen in the two data sets that are not in-source fragmentation, common contamination, or oxidative ionization product, and have at least one high quality MS/MS spectrum (Supplementary Table 1). Chemical composition analysis was performed with the maximum number of carbon, hydrogen, nitrogen, and oxygen of 50,100,5, and 15, respectively. Halides, sulfur, and phosphorous were not considered because they are not expected to be present in perfume, nor found in GCEI-MS analysis. All the assigned chemical compositions were below 3 ppm mass errors and they are the only chemical compositions possible within $5 \mathrm{ppm}$.

Except for two peaks, $m / z 192.0784\left(\mathrm{C}_{11} \mathrm{H}_{12} \mathrm{O}_{3}\right)$ and $m / z$ $234.1973\left(\mathrm{C}_{16} \mathrm{H}_{26} \mathrm{O}\right)$, all the other peaks are protonated pseudo-molecular ions. APPI is able to produce both protonated molecules and molecular radical cations. Depending on the experimental conditions, different abundance ratios of molecular radical cations versus protonated pseudomolecular ions were reported. For example, McEwen reported equally abundant molecular radical cations and protonated pseudo-molecular ions in GC-APPI-TOF of some perfume compounds ${ }^{10}$ while Revelsky and coworkers

Table 1. Tentatively identified perfume compounds in GC-APPI-LIT-Orbitrap through chemical composition analysis and MS/MS database search

\begin{tabular}{|c|c|c|c|c|c|}
\hline$m / z$ & Relative Intensity $^{a}$ & Mass Error (ppm) & Composition & Assignment $\mathrm{t}^{\mathrm{b}}$ & $\begin{array}{c}\text { Signal Improvement } \\
\text { by Dopant }^{\mathrm{c}}\end{array}$ \\
\hline 106.0861 & 1.4 & "-1.2 & 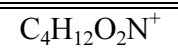 & Diethanol amine & - \\
\hline$\underline{147.0443}$ & $\underline{8.5}$ & 1.9 & $\underline{\mathrm{C}}_{2} \underline{\mathrm{H}}_{7} \underline{\mathrm{O}}_{2}^{ \pm}$ & $\underline{\text { Coumarin }}$ & $\underline{2.8}$ \\
\hline 150.1128 & 40.6 & 1.9 & $\mathrm{C}_{6} \mathrm{H}_{16} \mathrm{O}_{3} \mathrm{~N}^{+}$ & Triethanol amine & 0.17 \\
\hline$\underline{193.1589}$ & 17.2 & $\underline{0.9}$ & $\underline{\mathrm{C}}_{13} \underline{\mathrm{H}}_{21} \underline{\mathrm{O}}^{ \pm}$ & b-Ionone & $\underline{6.6}$ \\
\hline 225.1489 & 23.8 & 1.8 & $\mathrm{C}_{13} \mathrm{H}_{21} \mathrm{O}_{3}^{+}$ & Methyl Jasmonate & 1.0 \\
\hline 227.1645 & 17.4 & 1.3 & $\underline{\mathrm{C}}_{13} \underline{\mathrm{H}}_{23} \underline{\mathrm{O}}_{3}{ }^{ \pm}$ & Hedione & $\underline{28}$ \\
\hline
\end{tabular}

Italic underlined: Chemical compositions are also found in GC-EI-TOF and/or GC-EI-Q analysis.

${ }^{a}$ Relative intensities normalized against the base peak in the averaged MS spectrum over Rt of 1.4-13.5 min.

${ }^{\mathrm{b}}$ Times difference. Assignment is made through MS/MS database search.

${ }^{\mathrm{c}} \mathrm{Ratio}$ of the major peak in XIC with dopant over the corresponding peak without dopant. '-' indicates there is no reliable peak with dopant. 
Young Jin Lee, Erica A. Smith, and Ji Hyun Jun

Table 2. Chemical compositions of some perfume compounds with signal increase of more than twenty times with dopant spray

\begin{tabular}{|c|c|c|c|c|c|c|}
\hline \multirow{2}{*}{$m / z$} & \multirow{2}{*}{ Relative Intensity $^{a}$} & \multirow{2}{*}{ Mass Error (ppm) } & \multirow{2}{*}{ Composition } & \multirow{2}{*}{$\begin{array}{l}\text { Signal Improve- } \\
\text { ment by Dopant }\end{array}$} & \multicolumn{2}{|c|}{ Oxidative Ionization $(\%)^{\mathrm{c}}$} \\
\hline & & & & & Without Dopant & With Dopant \\
\hline 175.0755 & 68.1 & 0.7 & $\mathrm{C}_{11} \mathrm{H}_{11} \mathrm{O}_{2}^{+}$ & 86 & $115 / 438$ & $2.2 / 3.5$ \\
\hline$\underline{227.1645}$ & $\underline{29.0}$ & $\underline{1.3}$ & $\underline{\mathrm{C}}_{13} \underline{\mathrm{H}}_{23} \underline{\mathrm{O}}^{ \pm}$ & $\underline{28}$ & $\underline{8.6 / 12}$ & $\underline{0.1 / 0}$ \\
\hline 229.2164 & 86.0 & 0.8 & $\mathrm{C}_{14} \mathrm{H}_{29} \mathrm{O}_{2}^{+}$ & 166 & $0 / 0$ & $0 / 0$ \\
\hline 235.2057 & 199 & 0.2 & $\mathrm{C}_{16} \mathrm{H}_{27} \mathrm{O}^{+}$ & 530 & $709 / 837$ & $6.2 / 4.6$ \\
\hline$\underline{237.2216}$ & $\underline{46.1}$ & $\underline{1.2}$ & $\underline{\mathrm{C}}_{16} \underline{\underline{\mathrm{H}}} \underline{29} \underline{\mathrm{O}}^{ \pm}$ & $\underline{461}$ & $\underline{64 / 120}$ & $\underline{0.1 / 0}$ \\
\hline$\underline{259.2059}$ & $\underline{108}$ & $\underline{1.2}$ & $\underline{\mathrm{C}}_{18} \underline{\mathrm{H}_{27}} \underline{\mathrm{O}}^{ \pm}$ & $\underline{20}$ & $\underline{8.0 / 25}$ & $\underline{0.5 / 0.4}$ \\
\hline 271.2632 & $\underline{10.1}$ & $\underline{0.1}$ & $\underline{\mathrm{C}}_{17} \underline{\mathrm{H}}_{3 \underline{5}} \underline{\mathrm{O}}_{\underline{2}}^{ \pm}$ & $\underline{73}$ & $\underline{382 / 194}$ & $\underline{0.2 / 3.3}$ \\
\hline
\end{tabular}

Italic underlined: Chemical compositions are also found in GC-EI-TOF and/or GC-EI-Q analysis.

${ }^{a}$ Relative intensities normalized against the base peak in the averaged MS spectrum over Rt of 1.4-13.5 min, then scaled to the signal levels without dopant for comparison with Table 1.

${ }^{\mathrm{b}}$ Ratio of the major peak in XIC with dopant over the corresponding peak without dopant.

${ }^{\mathrm{c}}$ Yields of oxidative ionization products compared to protonated molecule: $\left[\mathrm{M}+\mathrm{O}+\mathrm{H}^{+}\right] /\left[\mathrm{M}+\mathrm{H}^{+}\right]$and $\left[\mathrm{M}+2 \mathrm{O}+\mathrm{H}^{+}\right] /\left[\mathrm{M}+\mathrm{H}^{+}\right]$.

reported predominance of protonated pseudo-molecular ions in GC-APPI-MS of butyldimethylsilylated amino acids. ${ }^{20}$ Microchip APPI was reported to preferentially produce protonated pseudo-molecular ions for androgenic steroids. ${ }^{5}$ In our experimental conditions, protonation seems to be dominant over radical cation formation.

For the comparison, GC-EI-Q and GC-EI-TOF analyses were performed for the same perfume sample. Combined, a total of 36 chemical compositions were tentatively identified for the two GC-EI-MS analyses as shown in Supplementary Table 2. In any of the data analyses, we did not use a retention time index for exact identifications of perfume compounds, because it is beyond the purpose of current study. Half the tentatively identified chemical compositions are mutually exclusive between GC-APPI-LIT Orbitrap and GC-EI-MS, suggesting the two techniques are complementary to each other. The primary difference is attributed to the difference in ionization efficiencies for different classes of compounds.

The presence of molecular ions, preferentially with chemical composition analysis, is often critical in EI-MS spectral interpretation. ${ }^{21}$ However, almost half the tentative identifications do not have molecular ions or have only weak ions $(<10 \%$ of base peak) in GC-EI-Q. For those molecular ions detected in GC-EI-TOF, chemical compositions were all matching with the corresponding library search results in $10 \mathrm{ppm}$ mass errors with the help of lockmass internal calibration and careful background removal. However, 7 out of 17 have two possible chemical compositions in $10 \mathrm{ppm}$ mass tolerance. The overall mass error for those tentatively identified compounds is $3.34 \pm 2.11 \mathrm{ppm}$ in GC-EI-TOF with internal calibration (Supplementary Table 2), while it is $1.32 \pm 0.65 \mathrm{ppm}$ in GC-APPI-Orbitrap without internal calibration (Supplementary Table 1).

\section{Structural assignments of MS/MS spectra}

A total of forty MS/MS spectra out of twenty six major chemical compositions in GC-APPI-LIT Orbitrap were manually searched against publicly available MS/MS databases: NIST 08 MS/MS database, Metlin (http:// metlin.scripps.edu/), and MassBank (http://www.massbank. $\mathrm{jp} /$ ). For the chemical compositions also identified in GCEI-Q or GC-EI-TOF, a literature survey was also performed to find the reported MS/MS spectra. Tentative chemical identification was made for six of them as summarized in Table 1. Most MS/MS spectra in the databases are acquired with a triple quadrupole mass spectrometer (QQQ) or QTOF. There are some major differences in MS/MS spectra between an ion trap mass spectrometer and QQQ or QTOF. Ion trap MS/MS produces mostly high mass fragments because of low mass cutoff and predominance of single fragmentations, while QQQ or Q-TOF produces a wide range of fragment ions particularly with low mass fragments from multiple activations and fragmentations. Careful comparison was made between our ion trap MS/MS spectra and those in the database; high to mid fragment ions are mostly used to determine the matching and the intensity differences are largely ignored. The database coverage is so poor that almost half the chemical compositions (12 out of 26) do not have any MS/MS spectra in any of the databases for the same chemical compositions.

Figure 4 shows an example of identified compounds, bIonone $\left(\mathrm{C}_{13} \mathrm{H}_{20} \mathrm{O}\right)$, a well-known perfume compound. Extracted ion chromatogram (XIC) of protonated b-Ionone constructed with $10 \mathrm{ppm}$ mass tolerance shows a single chromatographic peak at Rt of $9.2 \mathrm{~min}$, for both without and with dopant (Figure 4a and 4d). A series of oxidative precursor ions are present in the mass spectrum (Figure $4 \mathrm{~b}$ ): $m / z 209.1537\left(\mathrm{C}_{13} \mathrm{H}_{21} \mathrm{O}_{2}\right), m / z 225.1488\left(\mathrm{C}_{13} \mathrm{H}_{21} \mathrm{O}_{3}\right)$, and $m / z$ $241.1433\left(\mathrm{C}_{13} \mathrm{H}_{21} \mathrm{O}_{4}\right)$ with one, two, and three oxygen addition, respectively. The peak with $\mathrm{m} / \mathrm{z} 207.1382$ $\left(\mathrm{C}_{13} \mathrm{H}_{19} \mathrm{O}_{2}\right)$ is present in a significant amount which is regarded as the water loss of $m / z 225.1488\left(\mathrm{C}_{13} \mathrm{H}_{21} \mathrm{O}_{3}\right)$ by in-source CID; its dominance in the MS/MS spectrum of 

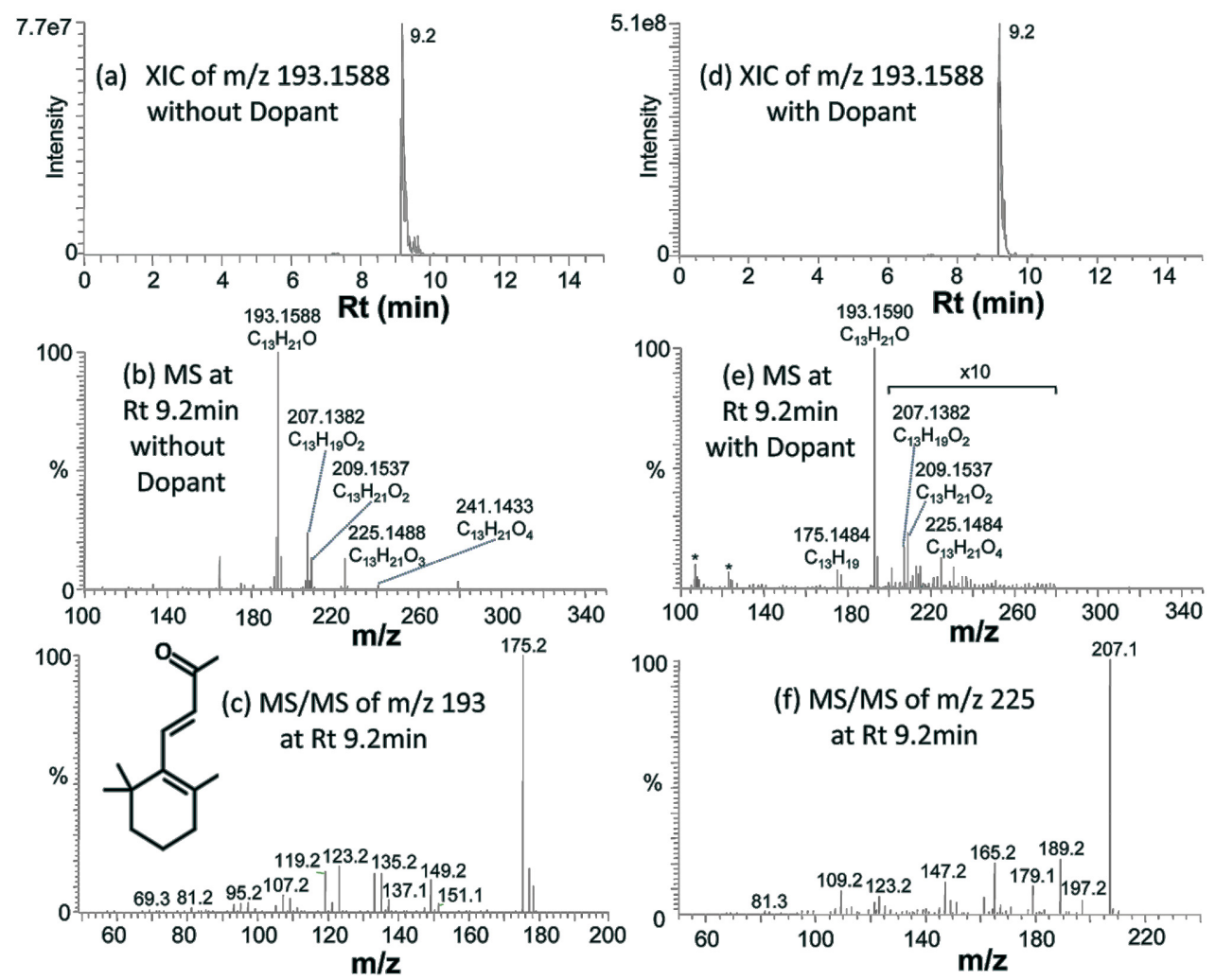

Figure 4. Identification of b-Ionone. XIC of $\mathrm{m} / z$ 193.1587 $\left(\mathrm{C}_{13} \mathrm{H}_{21} \mathrm{O}\right)$ (a) without and (d) with dopant spray. MS spectra at Rt $\sim 9.2 \mathrm{~min}$ (b) without and (e) with dopant spray shows the decrease of oxidative ionization with dopant. (c) MS/MS spec- trum of $m / z$ 193 matches that of b-Ionone. (f) MS/MS of $m / z 225(\mathrm{~m} / z 193+\mathrm{O})$ suggests $m / z 207.1382\left(\mathrm{C}_{13} \mathrm{H}_{19} \mathrm{O}_{2}\right)$ in MS (b or e) might be the water loss of $m / z 225.1488\left(\mathrm{C}_{13} \mathrm{H}_{21} \mathrm{O}_{3}\right)$. *: Back- ground ions produced by dopants.

$m / z 225$ (Figure 4f) supports this possibility. The MS/MS spectrum for protonated b-Ionone was not found in any of the three databases; however, it was reported by Prasain and co-workers. ${ }^{22}$ Their MS/MS spectrum was acquired with QQQ, but matches very well with Figure 4c; high abundance fragment ions are all observed in their MS/MS spectrum $(\mathrm{m} / \mathrm{z} 175,151,149,135,123,119,109,95,81$, and 69). b-Ionone was also identified in GC-EI-Q and GCEI-TOF, further supporting its identification.

Figure 5 shows the identification of two other wellknown perfume compounds, methyl jasmonate and methyl dihydrojasmonate (hedione). Hedione was detected in both GC-EI-Q and GC-EI-TOF, but methyl jasmonate was not. XIC of hedione ( $\mathrm{m} / \mathrm{z} 227.1645)$ shows a chromatographic peak at Rt $\sim 11.4$ min with 28 times signal improvement with dopant spray (Figure 5a and 5d). In the MS spectrum without dopant (Figure 5b), the three peaks with mass difference of $2 \mathrm{Da}$ at $m / z 223.1333\left(\mathrm{C}_{13} \mathrm{H}_{19} \mathrm{O}_{3}\right), 225.1489$ $\left(\mathrm{C}_{13} \mathrm{H}_{21} \mathrm{O}_{3}\right)$, and $227.1644\left(\mathrm{C}_{13} \mathrm{H}_{23} \mathrm{O}_{3}\right)$ have one oxygen addition products at $m / z 239\left(\mathrm{C}_{13} \mathrm{H}_{19} \mathrm{O}_{4}\right), 241\left(\mathrm{C}_{13} \mathrm{H}_{21} \mathrm{O}_{4}\right)$, and $243\left(\mathrm{C}_{13} \mathrm{H}_{23} \mathrm{O}_{4}\right)$ and two oxygen addition products at $m / z 255\left(\mathrm{C}_{13} \mathrm{H}_{19} \mathrm{O}_{5}\right), 257\left(\mathrm{C}_{13} \mathrm{H}_{21} \mathrm{O}_{5}\right)$, and $259\left(\mathrm{C}_{13} \mathrm{H}_{23} \mathrm{O}_{5}\right)$. The peak at $\mathrm{m} / \mathrm{z} 223$ and its oxidative precursor ions are regarded as the result of in-source water loss of $m / z 241$. It is not clear in Figure 4b whether $m / z 225$ (possibly methyl jasmonate) is a water loss of $\mathrm{m} / \mathrm{z} 243$, an oxidation of $\mathrm{m} / \mathrm{z}$ 227 (hedione). In the MS spectrum with dopant (Figure 4e), $\mathrm{m} / \mathrm{z} 227$ dominates the spectrum and its oxidation $(\mathrm{m} / \mathrm{z} 243)$ is only $0.1 \%$. Ion signals for $\mathrm{m} / \mathrm{z} 225$ and 241 are also greatly reduced with dopant; however, a significant amount still remains, $5.5 \%$ for $\mathrm{m} / z 225$ and $4 \%$ for $\mathrm{m} / z$ 241. This suggests methyl jasmonate $(\mathrm{m} / \mathrm{z} 225)$ might be present, although in much lower abundance, and its ionization is not favored with dopant unlike hedione. Further study is necessary to confirm its presence. MS/MS of $\mathrm{m} / \mathrm{z} 225$ (Figure 5f) correlates quite well with that of methyl jasmonate at Metlin and MS/MS of $\mathrm{m} / \mathrm{z} 227$ (Figure 5c) matches well with that of hedione at MassBank.

\section{Dopant co-spray}

Co-spraying dopant during GC-APPI-MS analysis has two advantages for some compounds like b-Ionone and hedione. As can be seen from the Y-scales of Figure $4 \mathrm{a}$ and $4 \mathrm{~d}$ and Figure $5 \mathrm{a}$ and $5 \mathrm{~d}$, the ion signal was improved by 6.6 times for b-Ionone and 28 times for hedione by spraying dopant. In addition, oxidative ionization is significantly reduced in its MS spectrum. b-Ionone, for example, has ten times lower relative abundances for $m / z 207$ (+O), 209 

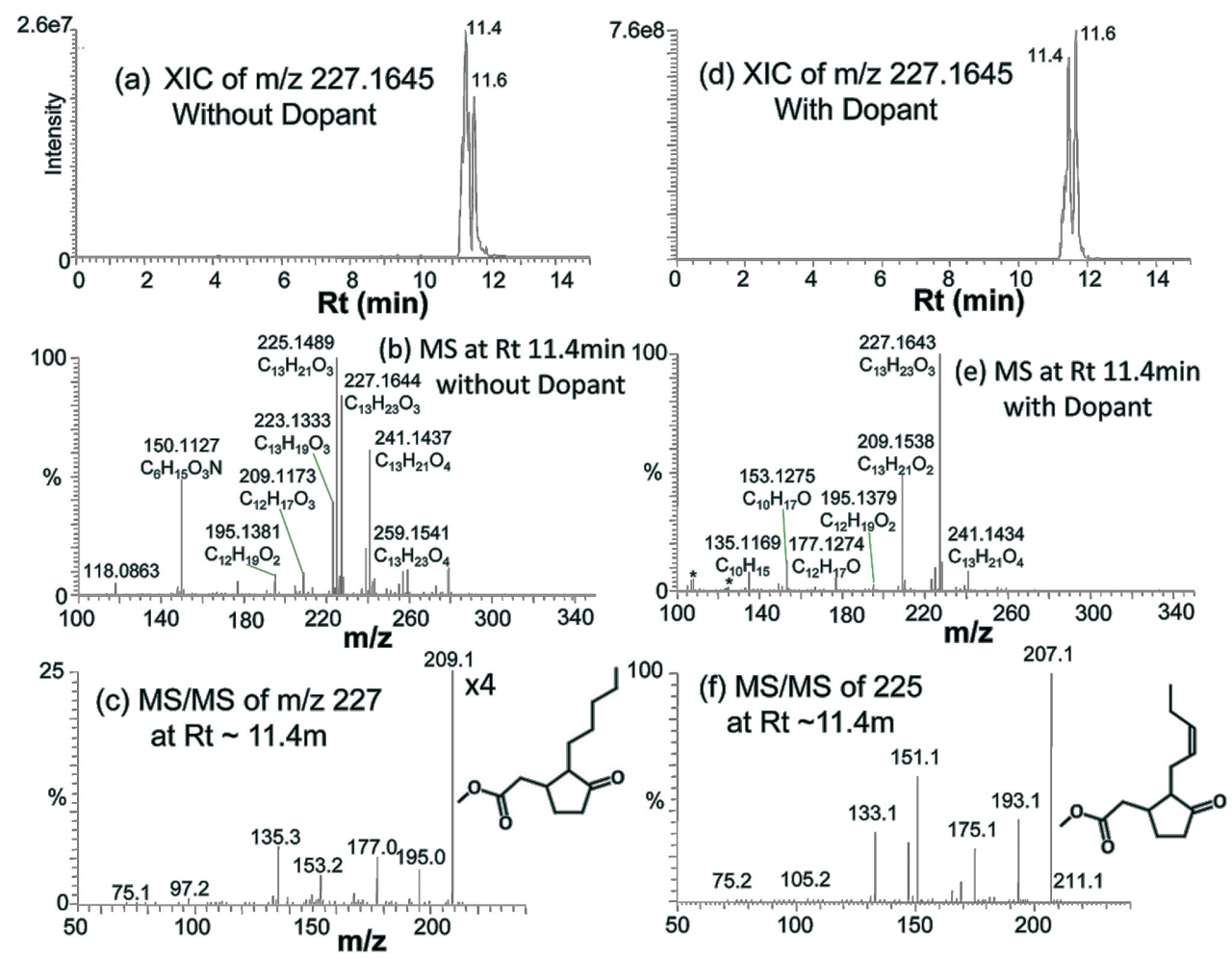

Figure 5. Identification of hedione and methyl jasmonate. XIC of $m / z 227.1645\left(\mathrm{C}_{13} \mathrm{H}_{23} \mathrm{O}_{3}\right)$ (a) without and (d) with dopant spray. MS spectra at Rt $\sim 11.4 \mathrm{~min}$ (b) without and (e) with dopant spray. MS/MS spectra of (c) $\mathrm{m} / \mathrm{z} 227$ and (f) $\mathrm{m} / z 225$ at Rt $\sim 11.4 \mathrm{~min}$ match those of hedione and methyl jasmonate, respectively. *: Background ions produced by dopants.

$\left(+2 \mathrm{O}-\mathrm{H}_{2} \mathrm{O}\right), 225(+2 \mathrm{O})$, and $241(+3 \mathrm{O})$ with dopant than those without dopant (Figure $4 \mathrm{e}$ vs. Figure $4 \mathrm{~b}$ ). The same trend can also be found for hedione in Figure $5 \mathrm{e}$ vs. Figure 5b. Figure 6 demonstrates how significantly dopant can enhance ionization and detection of some compounds. XIC of $m / z 229.2164\left(\mathrm{C}_{14} \mathrm{H}_{29} \mathrm{O}_{2}\right)$ shows a clear peak both without and with dopant (Figure $6 \mathrm{a}$ and $6 \mathrm{~b}$ ). Its ion signal, however, is very low in MS spectrum without dopant (Figure 6c), and MS/MS spectrum was not acquired. Even if MS/MS were acquired, it could have been contaminated by the interfering peak near-by with the mass difference of only $0.02 \mathrm{Da}\left(\mathrm{m} / \mathrm{z} 229.1949\left(\mathrm{C}_{17} \mathrm{H}_{25}\right)\right)$ as shown in the inset spectrum of Figure $6 \mathrm{c}$. With dopant spray, the peak intensity is enhanced by 166 times (scale difference between Figure $6 \mathrm{a}$ and $6 \mathrm{~b}$ ) and the mass peak is now clearly distinguishable in MS spectrum (Figure 6d) and MS/MS spectrum was successfully acquired (data not shown).

Table 2 summarizes some major compounds with at least twenty times signal improvement by spraying dopant. Signal improvement was up to two orders of magnitude and oxidative ionization was all significantly reduced. There is a close correlation between the ion signal enhancement and the oxidation reduction induced by dopant spray. In general, signal enhancement is more significant when there is higher oxidation without dopant. However, the amount of oxidation reduction does not quantitatively correspond to the signal improvement. In addition, some other ions, such as $m / z 229.2164$, do not have detectable amount of oxidation but it has 166 times of signal improvement by dopant.

Dopant spray has a few limitations in the application to GC-APPI-MS. First, new background ions show up with significant intensities; a series of ions at $\mathrm{m} / \mathrm{z}$ 70-140 marked as stars $(*)$ in Figure $4 \mathrm{e}, 5 \mathrm{e}$, and $6 \mathrm{~d}$. They are mostly toluene and its oxidation/fragmentation products such as $\mathrm{C}_{6} \mathrm{H}_{7}^{+}, \mathrm{C}_{7} \mathrm{H}_{7}^{+}, \mathrm{C}_{6} \mathrm{H}_{7} \mathrm{O}^{+}, \mathrm{C}_{7} \mathrm{H}_{7} \mathrm{O}^{+}, \mathrm{C}_{7} \mathrm{H}_{8} \mathrm{O}^{+*}, \mathrm{C}_{7} \mathrm{H}_{7} \mathrm{O}_{2}^{+}$, and $\mathrm{C}_{7} \mathrm{H}_{8} \mathrm{O}_{2}^{+}$. Another disadvantage is it dramatically decreases some ion signals, as notable from the disappearance of most ion signals for Rt below $8 \mathrm{~min}$ in Figure 2 with dopant spray. Lastly, in-source fragmentation seems to increase by spraying dopant. In-source water loss fragmentation often found in our precursor spectra is enhanced by spraying dopant; i.e., $m / z 175$ from water loss of $m / z 193$ in Figure 4e versus Figure $4 \mathrm{~b}$. It is even prominent in MS/MS of hedione. Fragments of hedione, such as $m / z 209,195,177$, 153, and 135 (Figure 4c), have much higher signals in the precursor spectrum with dopant (Figure 4e) than those without dopant (Figure 4b). Hedione seems to be exceptional coming from the instability of protonated ester ions and excess internal energy provided by toluene-induced protonation. A systematic study is needed to further understand dopant assisted ionization and minimize dopant assisted insource fragmentation. 


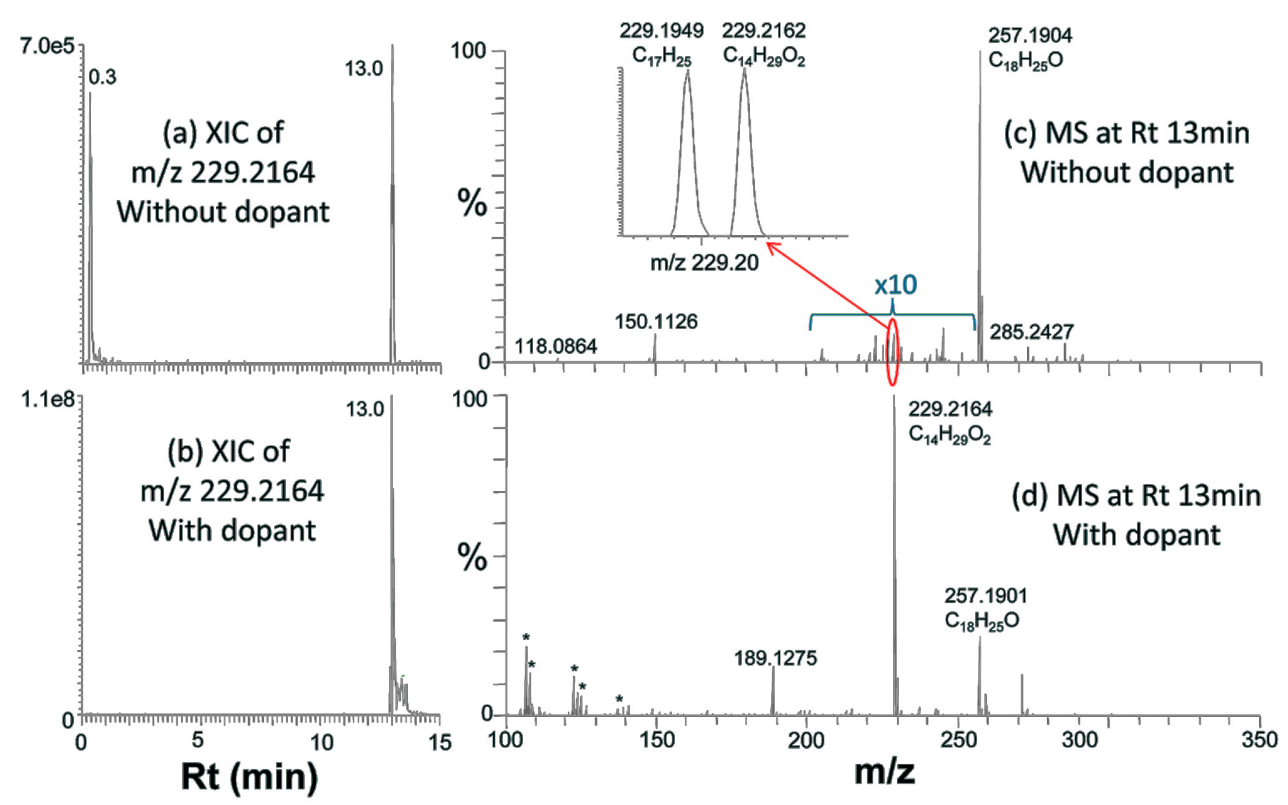

Figure 6. Signal improvement of $m / z 229.2164\left(\mathrm{C}_{14} \mathrm{H}_{29} \mathrm{O}_{2}\right)$ with dopant. XIC of $m / z 229.2164$ (a) without and (b) with dopant. In MS spectrum without dopant (c), $\mathrm{m} / \mathrm{z} 229.2164$ is barely seen and has a significant peak near-by that will interfere MS/MS spectral acquisition. (d) MS spectrum with dopant shows a clear peak at $m / z$ 229.2164. *: Background ions produced by dopants.

\section{Conclusion}

We have developed a new approach for the analysis of complex volatile compounds, GC-HRMS/MS, using GCAPPI-LIT Orbitrap. Chemical composition analysis of precursor ions followed by an MS/MS spectral search was successfully demonstrated for the analysis of a complex perfume sample. This approach is complementary to the conventional GC-EI-MS analysis, improving the confidence in identification of the compounds with both methods and increasing the chance to identify low abundance co-eluting compounds. Above all, it is compatible with atmospheric pressure ionization sources designed for LC-MS/MS and easily adaptable to high-end mass spectrometers without need of significant instrument modifications. A few hurdles need to be overcome for this approach to become useful as a tool for high throughput volatile compound analysis. Experimental conditions need to be optimized to minimize in-source oxidation and fragmentation. The most critical limitation is the insufficient coverage of the current MS/MS databases, which should be eventually overcome as the public MS/MS database size increases and/or by the success of a priori MS/MS spectral prediction. Specifically, Fragmentation Library ${ }^{\mathrm{TM}}$ built with an extensive literature survey seems very promising. ${ }^{19}$

APPI-LIT Orbitrap is best suited for the proposed GCHRMS/MS approach. Its scan speed is not as fast as TOF, but is comparable to that of quadrupole MS, and it provides sufficient data points for each chromatographic peak. Its reliable mass accuracy is the greatest advantage over TOF
MS and could provide 3-4 ppm mass accuracy without any internal calibration. If needed, lock-mass calibration could be utilized to achieve 1-2 ppm mass accuracy. ${ }^{4,23}$ As suggested by Kind and Fiehn, even 1 ppm mass accuracy might not be sufficient to assign a unique chemical composition when more chemical elements are in consideration. ${ }^{24}$ Isotope peak ratios and other criteria could be utilized to enhance the confidence of elemental composition analysis. ${ }^{24,25}$ The most recent Orbitrap mass spectrometer, such as QExactive (12 Hz, mass resolution of up to 140,000), provides much faster scan speed and better mass resolution. We envision GC-APPI-QTOF could also be efficiently used for the proposed GC-HRMS/MS approach because of its fast scan speed, decent mass accuracy, wide range of fragment ions (thus, better compatibility with the current databases), and, most of all, accurate tandem mass spectra.

Over the half century of GC-MS history, the analysis of complex volatile compounds largely relied on high resolution gas chromatographic separation and GC-EI-MS spectral library search. The fundamental limitation of the traditional approach is obvious, especially for extremely complex mixtures with wide dynamic ranges. We are hopeful the proposed approach would become a powerful tool to complement the current EI-MS based approach and resolve the current bottleneck.

\section{Acknowledgements}

This work is supported by grants from Iowa State University and Ames Laboratory, U.S. DOE. E. A. S. 
acknowledges a Graduate Assistance in Areas of National Need (GAANN) fellowship from the U.S. Department of Education. We acknowledge Steve Veysey in Chemical Instrument Facility for his assistance in running GC-EI-TOF.

\section{Supplementary Material}

Supplementary Tables and Figures associated with this article may be found in the online version.

\section{References}

1. Kitson, F. G.; Larsen, B. S.; McEwen, C. N. Gas Chromatography and Mass Spectrometry, a Practical Guide; Academic Press, New York, 1996.

2. Veriotti, T.; Sacks, R. Anal. Chem. 2001, 73, 4395.

3. Luo, Z.; Heffner, C.; Solouki, T. J. Chromatogr. Sci. 2009, 47, 75.

4. Peterson, A. C.; McAlister, G. C.; Quarmby, S. T.; GriepRaming, J.; Coon, J. J. Anal. Chem. 2010, 82, 8618.

5. Luosujärvi, L.; Haapala, M.; Thevis, M.; Saarela, V.; Franssila, S.; Ketola, R. A.; Kostiainen, R.; Kotiahoa, T. J. Am. Soc. Mass Spectrom. 2010, 21, 310.

6. Stein, S. E. J. Am. Soc. Mass Spectrom.1994, 5, 316.

7. Horning, E. C.; Horning, M. G.; Carroll, D. I.; Dzidic, I.; Stillwell, R. N. Anal. Chem. 1973, 45, 936.

8. Mitchum, R. K.; Korfmacher, W. A.; Moler, G. F.; Stalling, D. L. Anal. Chem. 1982, 54, 719.

9. McEwen, C. E.; McKay, R. G. J. Am. Soc. Mass Spectrom. 2005, 16, 1730.

10. McEwen, C. E. Int. J. Mass Spectrom. 2007, 259, 57.

11. Bristow, T.; Harrison, M.; Sims, M. Rapid Commun. Mass Spectrom. 2010, 24, 1673.

12. Carrasco-Pancorbo, A.; Nevedomskaya, E.; ArthenEngeland, T.; Zey, T.; Zurek, G.; Baessmann, C.; Deelder,
A. M.; Mayboroda, O. A. Anal. Chem. 2009, 81, 10071.

13. Portolés, T.; Sancho, J. V.; Hernández, F.; Newtonb, A.; Hancock, P. J. Mass. Spectrom. 2010, 45, 926.

14. Müller, A.; Düchting, P.; Weiler, E. W. Planta 2002, 216, 44.

15. Haas, W.; Faherty, B. K.; Gerber, S. A.; Elias, J. E.; Beausoleil, S. A.; Bakalarski, C. E.; Li, X.; Villen, J.; Gygi, S. P. Mol. Cell. Proteomics 2006, 5, 1326.

16. Makarov, A.; Denisov, E.; Lange, O.; Horning, S. J. Am. Soc. Mass Spectrom. 2006, 17, 977.

17. Fjeldsted, J. Time-of-Flight Mass Spectrometry, Technical Overview; Report 5989-0373EN, Agilent Technologies: 2003.

18. Heinonena, M.; Rantanena, A.; Mielikäinena, T.; Pitkänena, E.; Kokkonenb, J.; Rousua, J. German Conference on Bioinformatics $G C B$, p. 40-53, 2006.

19. Lutisan, J.; Huang, Y.; Nikiforov, A.; Suchy, M.; Mistrik, R. ThOEam 09:30, May 27, 58th ASMS Conference on Mass Spectrometry, Salt Lake City, Utah, May 23-27, 2010.

20. Revelsky, I. A.; Yashin, Y. G.; Sobolesvsky, T. G.; Revelsky, A. I. Eur. J. Mass Spectrom. 2003, 9, 497.

21. McLafferty, F. W.; Turecek, F.: Interpretation of Mass Spectra, 4th ed., University Science Books, Mill Valley, 1993.

22. Prasain, J. K.; Moore, R.; Hurst, J. S.; Barnes, S.; Frederik, F. J. G. M. J. Mass Spectrom. 2005, 40, 916.

23. Olsen, J. V.; de Godoy, L. M. F.; Li, G.; Macek, B.; Mortensen, P.; Pesch, R.; Makarov, A.; Lange, O.; Horning, S.; Mann, M. Mol. Cell. Proteomics 2005, 4, 2010.

24. Kind, T.; Fiehn, O. BMC Bioinformatics 2006, 7, 234.

25. Kind, T.; Fiehn, O. BMC Bioinformatics 2007, 8, 105.

26. Olsen, J. V.; Macek, B.; Lange, O.; Makarov, A.; Horning, S.; Mann, M. Nat. Methods 2007, 4, 709. 